\title{
Polarization measurements on SUMI's TVLS gratings
}

\author{
K. Kobayashi, E. A. West, J. M. Davis and G. A. Gary \\ NSSTC/NASA, Marshall Space Flight Center, AL 35812
}

\begin{abstract}
We present measurements of toroidal variable-line-space (TVLS) gratings for the Solar Ultraviolet Magnetograph Investigation (SUMI), currently being developed at the National Space Science and Technology Center (NSSTC). SUMI is a spectro-polarimeter designed to measure magnetic fields in the solar chromosphere by observing two UV emission lines sensitive to magnetic fields, the CIV line at $155 \mathrm{~nm}$ and the MgII line at $280 \mathrm{~nm}$. The instrument uses a pair of TVLS gratings, to observe both linear polarizations simultaneously. Efficiency measurements were done on bare aluminum gratings and aluminum/ $\mathrm{MgF}_{2}$ coated gratings, at both linear polarizations.
\end{abstract}

Keywords: UV polarization, Solar Magnetograph, Sun, Sounding Rocket, Toroidal gratings, VLS gratings, double Wollaston polarizer

\section{INTRODUCTION}

The Solar Ultraviolet Magnetograph Investigation, SUMI, has been reported in several papers since this program began in $2000^{1,2,3}$. The emphasis in this paper will be to reference and summarize the test results presented in those papers while documenting the new tests of the last component that has been developed for SUMI, the toroidal variable-linespace (TVLS) gratings with emphasis on their polarization characteristics.

Section 2 will review the scientific goals of SUMI and why this mission is important in understanding the 3D structure of the magnetic field on the Sun. Section 2 will also provide a brief description of the SUMI sounding rocket. The test facility at the NSSTC will be described in Section 3. Section 4 will describe the polarization measurements of the TVLS gratings and compare those measurements with those publish earlier ${ }^{4}$. Finally Section 5 will summarize the polarization measurements made on the SUMI optics.

\section{DESCRIPTION OF SUMI}

This section will describe the importance of direct magnetic field measurements at various heights in the solar atmosphere and how the SUMI sounding rocket program plans to verify that measurements in the transition region are possible. While the SUMI sounding rocket program is a proof-of-concept mission, the goal for this mission is to obtain high resolution CIV spectro-polarimetry data of a simple large sunspot so that this data can be compared to the theoretical models and NLTE radiative codes that are being developed for this important transition region line e $^{1,2}$. While a large simple sunspot may not produce the polarization maps required to understand complicated structures in the transition region, SUMI's flight is an essential step in understanding the CIV measurements and the modeling require to connect the photospheric magnetic field to the magnetic fields observed in the transition region.

\subsection{Magnetic field measurements in the transition region}

The problem of how energy, stored in the solar magnetic field, is released to heat the corona and drives the dynamic phenomena of the outer atmosphere (flares and coronal mass ejections) remains unsolved. Neither theoretical or numerical models nor observations are able to provide a conclusive story. We have learned that a substantial fraction of this energy propagates outward into the interplanetary medium in the form of radiation, the solar wind, energetic particles, and shocks that create, control and modify the structure of the interplanetary medium. These products constitute space weather and have been shown to affect the immediate terrestrial environment, to create hazards for terrestrial and space assets, and will, in the future, be a major concern for astronauts traveling through interplanetary 
space and working on the lunar surface. The solar input is the logical starting point for any model that describes and predicts space weather. Therefore understanding the processes that contribute to both the steady and transient release of magnetic energy into the solar atmosphere is a crucial element of these studies.

To understand the fundamental processes that lead to the explosive release of magnetic energy in solar flares and coronal mass ejections (CMEs), it is necessary to observe and infer the topology of the magnetic field and how it evolves prior to and during the energy release process. The crucial and missing element is the ability to understand and quantitatively describe the transition layer where the field is no longer pressure-dominated as in the photosphere. The need to understand the energy release processes was identified two decades ago, and was reaffirmed as the major conclusion of a workshop on the definition of the scientific goals for "Beyond Solar-B" (Moore, Davis, and Hathaway, 2001) ${ }^{6}$. To gain this knowledge will require measuring the full vector field at several different heights in the solar atmosphere from the photosphere to the upper transition region where the field becomes force free. The vector field in the photosphere and low chromosphere has been measured with ground based instruments. Space-based instruments such as the Michelson Doppler Interferometer (MDI) on SOHO has demonstrated that continuous, distortion free, observations of the longitudinal magnetic field from space is very important in our understanding of the Sun's magnetic field and the Solar Optical Telescope (SOT) on Hinode and the Helioseismic and Magnetic Imager (HMI) on SDO ( 2008) will extend our space based observations to include photospheric vector magnetic fields. Although these high resolution observations will greatly advance our knowledge of the behavior of the magnetic field in the photosphere, they will shed little light on how the field is behaving higher in the atmosphere as the field transitions to a nearly force free state. This requires measurement of the field using spectral lines that are formed at temperatures above $20,000^{\circ} \mathrm{K}$. The two spectral lines we have chosen for SUMI are MgII and CIV. These lines occur in the far ultraviolet and are inaccessible from the ground. Together with FeI, NaI and CaII, they form a quintet of magnetically sensitive lines chosen for a future mission called the Magnetic Transition Region Probe (MTRAP) ${ }^{7}$. The demonstration that the MgII and CIV lines can meet the requirements for the force free region is a prerequisite for this mission and is one of the objectives of the Solar Ultraviolet Magnetograph Investigation (SUMI).

Some success has been achieved in the characterization of the full vector field in the photosphere, where $\beta$, the ratio of the gas pressure to the magnetic pressure, is $>1$. At higher levels in the atmosphere (Figure 1$)^{3}$ where $\beta<1$, the magnetic field (through the Lorentz force) controls the structure and dynamics of the solar atmosphere, and rapid changes in its structure can produce energetic events. However, observations of the magnetic field at these higher levels have proven to be difficult, placing a serious limitation on our understanding of the physical processes actually occurring there.

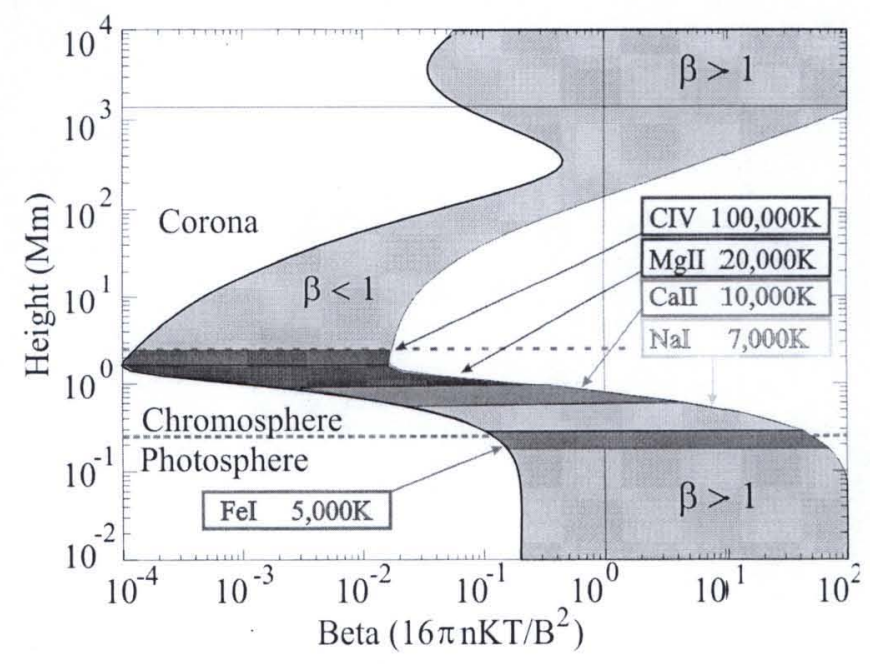

Figure 1. Ratio of gas pressure to magnetic pressure $(\beta)$ as a function of height, for an assumed range of photospheric magnetic field strength of 100 to $2500 \mathrm{G}$ (Garv. 2001). 


\subsection{Goals for SUMI sounding rocket program}

By the early nineties it was apparent that vector field measurements in the upper chromosphere and transition region were needed, even though this was viewed as a difficult if not impossible task. The Ultraviolet Spectrometer and Polarimeter (UVSP) instrument on board the Solar Maximum Mission (SMM) was the first space-based instrument to try to map and understand polarization in the transition region. The UVSP observations of MgII suggested that this line was a potential candidate for the upper chromosphere. Although UVSP attempted measurements of the CIV line, photon statistics and the loss of the CIV line early in the mission failed to produce conclusive evidence that this measurement was possible. The scientific value of these observations has been acknowledged ${ }^{10,11}$ and the SUMI sounding rocket program was born from development programs $\mathrm{s}^{1,2,3}$ to improve the overall wavelength and polarization efficiency of the CIV $(155 \mathrm{~nm})$ measurement while including the MgII measurements in its observing program. While these development programs were successful, accommodating the dual-line instrument in a sounding rocket envelope has been challenging.

CIV has always been the driving force in the development of SUMI. While this line is formed primarily in the transition region which simplifies its interpretation, the magnetic sensitivity $\left(\lambda^{2} g\right.$, where $\lambda$ is the wavelength and $g$ is Lande $g$ factor) is low when compared to lines in the visible and infrared wavelengths. Table 1 compares the SUMI lines with lines that are being used or proposed for future space-based telescopes. Certainly the results from SUMI will impact future missions as scientist try to understand the 3-D structure of the Sun's magnetic field.

\subsection{Description of SUMI sounding rocket}

A solar telescope design must solve the thermal problems associated with direct solar viewing. The simplest solution is a Gregorian telescope with a field stop between the primary and secondary mirrors. While this reduces the thermal load on the secondary by rejecting the unwanted light, the disadvantages to this approach are a limited field of view, a longer optical path, and a larger secondary for on-axis designs, which decreases the effective collecting area of the telescope. The SUMI approach for decreasing the thermal load on the secondary mirror is to use a Ritchey-Chretien telescope design with special dielectric coatings (Figure 3), applied to the front surfaces of both the primary and secondary mirrors. These coatings reflect only the narrow wavelength ranges around the CIV and MgII emission lines which results in a "cold mirror", i.e., a "self-filtering" telescope. The rear surface of the primary mirror is figured and has an aluminum coating that reflects the unwanted radiation back through the telescope. The advantages of this design are: the field of view is not restricted (whole Sun could be imaged by a slit jaw camera) and, for a given instrument length, the smaller telescope size allows a larger spectrograph improving the wavelength resolution.

The first section following the telescope is the polarimeter, spectrograph slit and slit jaw camera. The polarimeter consists of a $\mathrm{MgF}_{2}$ waveplate and a double Wollaston analyzer. Due to the low photon flux at CIV and the weak linear polarization levels, the waveplate was designed to measure circular polarization at CIV $\left(270^{\circ}\right.$ retardance at $\left.1550 \AA\right)$ and the full Stokes vector at $\mathrm{MgII}\left(131^{\circ}\right.$ retardance at $\left.2800 \AA\right)$. 


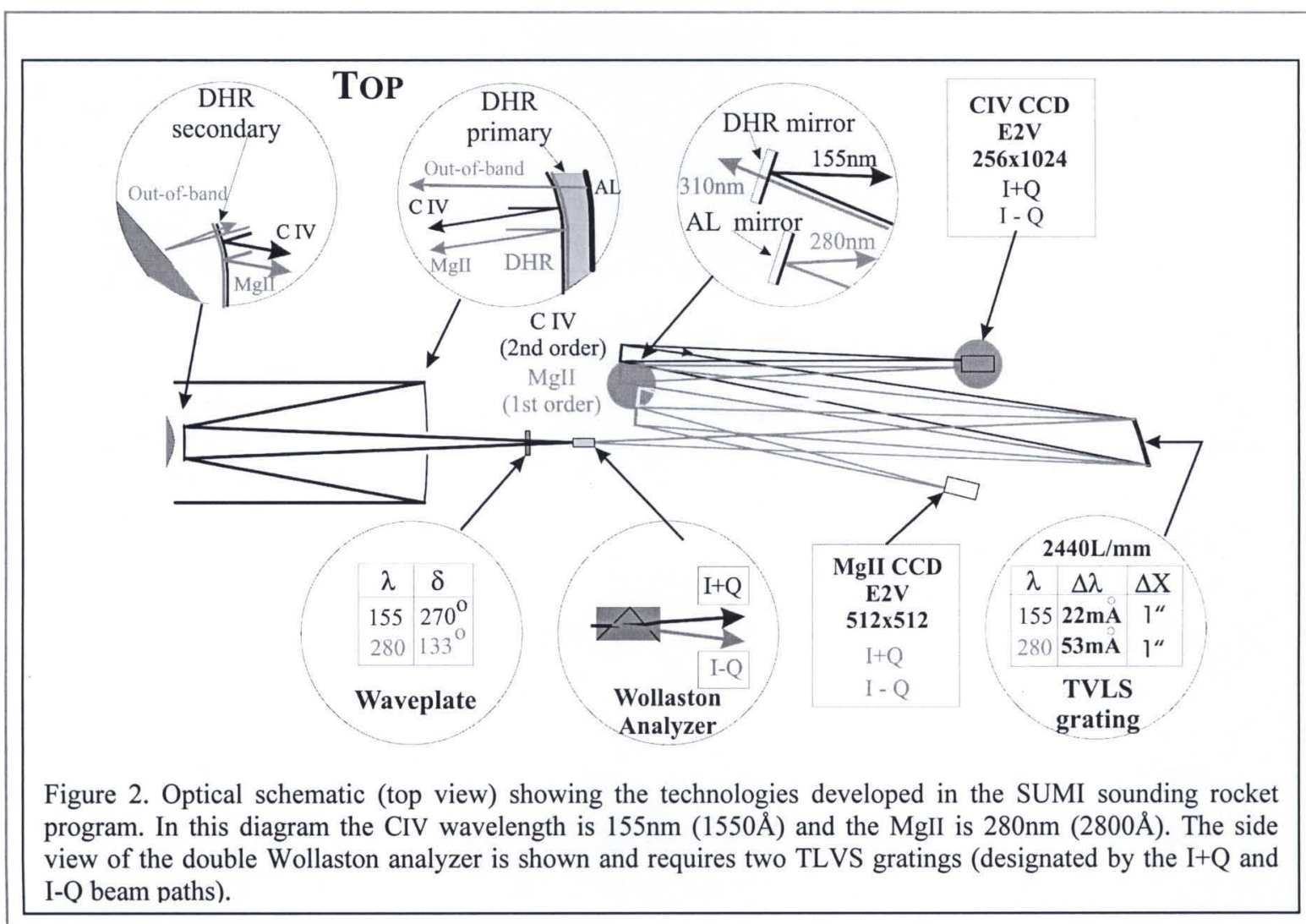

A dual-beam analyzer is very important in transition region magnetic field measurements since the CIV emission is a very dynamic. Without simultaneous measurements of orthogonal polarizations, intensity crosstalk could create false signals in the magnetic field measurements. Since both polarizations are observed, the total transmission is much higher than traditional reflective polarizers even at $\mathrm{CIV}$ which is near the cutoff

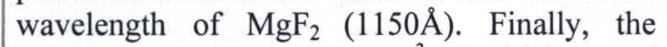
polarization resolution $\left(<10^{-3}\right)$ is higher and covers a larger wavelength range than traditional reflective analyzers which is very important for

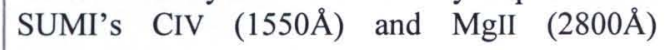
measurements.

Sensitivity of the spectrograph gratings to the polarization exiting the double Wollaston has always been considered ${ }^{8}$. In our initial concept a quarterwave plate could be placed between the double Wollaston and gratings to convert the exiting linear polarization to circular, minimizing the difference in reflectivity if the gratings were very sensitive to polarization such as the grating in Figure 12. While the quarterwave plate is a solution, it is also another

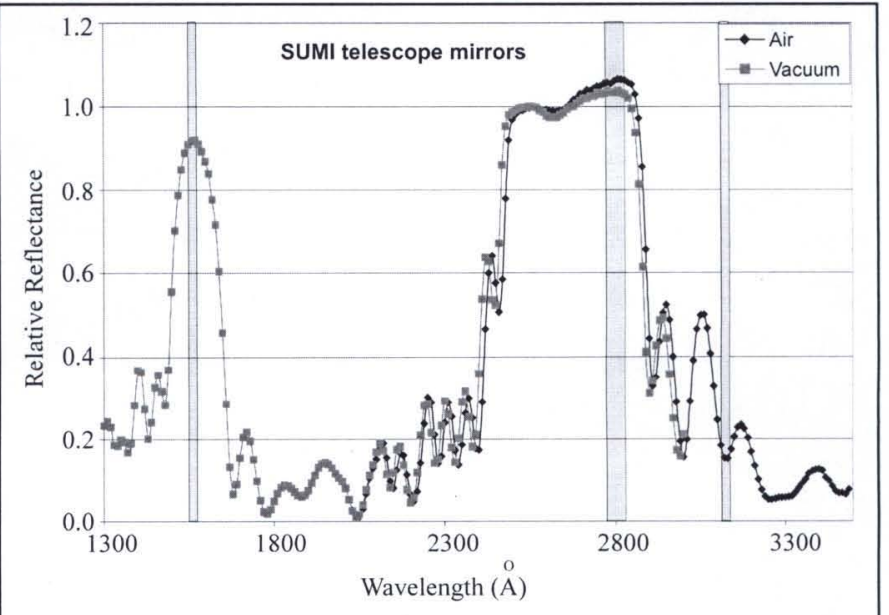

Figure 3. Dielectric High Reflectance (DHR) coating on SUMI primary and secondary telescope mirrors. These

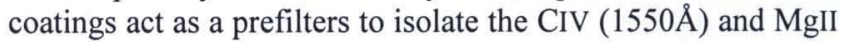
$(2800 \AA)$ lines. The MgII reflectivity was tuned to minimize the $1^{\text {st }}$ order reflectance of $3100 \AA$ into the $2^{\text {nd }}$ order CIV measurement. 


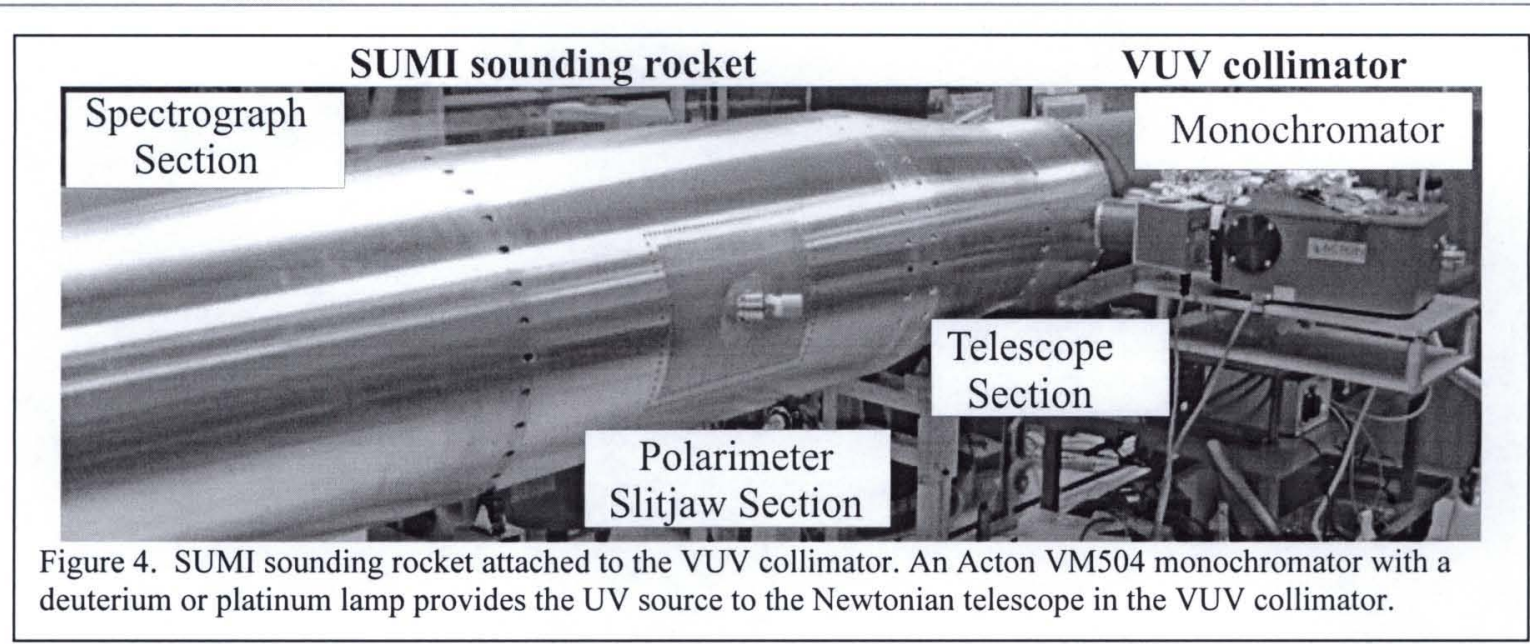

"absorption loss" at CIV. Therefore, selecting a grating that has a high reflectivity for the horizontal polarization and a second grating for the vertical polarization simplifies the optical design and removes an optical element that will absorb CIV. This will be discussed in greater detail in Section 4.

The CIV fold mirrors following the TVLS gratings have the same DHR coating that reflects the $1550 \AA$ wavelength band (Figure 3) but not the $2800 \AA$ DHR coating. With this additional reflection the total reflectivity at $3100 \AA$ is $<0.1 \%$. The MgII fold mirrors use a standard aluminum/ $/ \mathrm{MgF}_{2}$ coating.

The SUMI cameras are developed around the E2V back illuminated, bare silicon technology. This technology enhances the UV quantum efficiency but was limited to certain CCD structures since this technology was not part of E2V's standard product

Table 1. Minimum requirements and current spectrograph resolution. These requirements were based on SUMI's original scientific goals.

\begin{tabular}{|c|c|c|}
\hline Description & $\begin{array}{c}\text { Minimum } \\
\text { requirement }\end{array}$ & $\begin{array}{c}\text { TVLS } \\
\text { design }\end{array}$ \\
\hline Slit field of view $(\operatorname{arcsec})$ & $>120$ & $\begin{array}{c}150 \\
(210)\end{array}$ \\
\hline & & \\
\hline CIV & & \\
\hline Wavelength resolution $(\mathrm{m} \AA)$ & $<50$ & 22 \\
\hline Spatial resolution(arcsec) & $<2$ & 1.2 \\
\hline & & \\
\hline MgII & & \\
\hline Wavelength resolution $(\mathrm{m} \AA)$ & $<80$ & 53 \\
\hline Spatial resolution $(\operatorname{arcsec})$ & $<1$ & 1.0 \\
\hline
\end{tabular}

line. For the slitjaw camera, and the MgII cameras (Figure 2) where high speed shuttering is required, a frame transfer $512 \times 512$ CCD array with $13 \mu \mathrm{m}$ pixels was selected. The CIV camera uses a full frame $1024 \times 256$ CCD array with 26 $\mu \mathrm{m}$ pixels. To simplify the electrical wiring between the cameras and the data system, a USB 2.0 interface was selected. For ground-based testing and software development, a $\mathrm{MgF}_{2}$ window is mounted in front of all of the cameras. The measured quantum efficiency $(\mathrm{QE})$ for these cameras is $>60 \%$.

Finally, all of the optical components have been fabricated and tested. The sounding rocket is being assemble (Figure 4) and will launch in the summer of 2008. The next two sections will describe the test facility at the NSSTC and the polarization measurements made on the SUMI TVLS gratings. 


\section{DESCRIPTION OF TEST FACILITY}

The vacuum ultraviolet (VUV) facility (Figures 5 and 6) was originally developed by the Space Plasma Physics Branch at Marshall Space Flight Center to test the Ultraviolet Imager for the International Solar Terrestrial Physics Mission and for the Wide Imaging Camera for the IMAGE Mission. The system was moved to the NSSTC in 2000 and has been upgraded to measure the polarization properties of the SUMI telescope and polarimeter. The facility is capable of measuring the optical properties (transmittance, reflectance and polarization) of test samples in the wavelength range from $115 \mathrm{~nm}$ through the visible. To minimize exposure of test optics to contamination, a serious concern for VUV optics, the spectrophotometric system uses a stainless steel vacuum chamber that is maintained in a class $10 \mathrm{~K}$ clean room. The cryogenic hydrocarbon-free pumping system operates with a base pressure in the $10^{-7}$ torr range. The source for VUV measurements is a highpressure arc discharge deuterium lamp. Because of its continuum output in the $115-370 \mathrm{~nm}$ range, this source can be used to scan the reflection, transmission and polarization characteristics of high resolution dielectric mirrors and polarization optics in this wavelength band. A $0.2 \mathrm{~m}$ vacuum monochromator, with a concave holographic grating (1200 lines $/ \mathrm{mm})$ coupled to a $76.2 \mathrm{~cm}$ focal length collimating UV enhanced mirror system, produces a $10.2 \mathrm{~cm}$ diameter monochromatic collimated incident beam. This facility uses $\mathrm{MgF}_{2}$ birefringent analyzers (double Wollaston and Rochon) and zero-order $\mathrm{MgF}_{2}$ waveplates, to calibrate and measure the polarization properties. Vacuum compatible

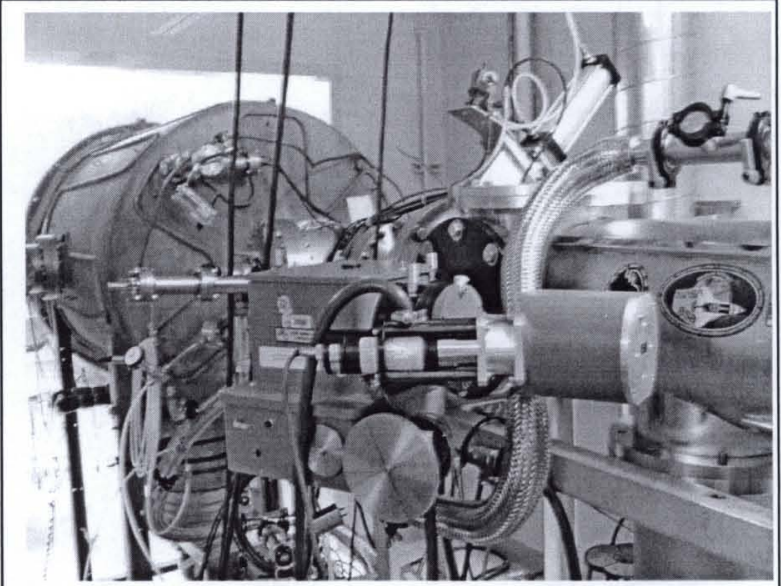

Figure 5. Picture of UV test chamber at the NSSTC

linear and rotational stages are used to position the detectors, optical components and test samples during the calibration and testing process. Labview is the software that is normally used to automate the positioning, light measurement and data archiving. Several UV sensitive photodiodes and solar blind PMTs are available for use depending on the test requirements. The entrance and exit to the monochromator have a three position filter wheel which allows optics to be inserted to eliminate $1^{\text {st }}$ order contamination (i.e. entrance slit: $155 \mathrm{~nm}$ into $310 \mathrm{~nm}$ measurements) and for optical tests in the main chamber using frosted $\mathrm{MgF}_{2}$ plates and test patterns (exit slit). Finally, a platinum source is used to calibrate the wavelength drives of the NSSTC UV monochromators since those measurements can be compared to the online platinum data found at the National Institute Standards and Technology (NIST) web site (http://physics.nist.gov/PhysRefData/platinum/contents.html).

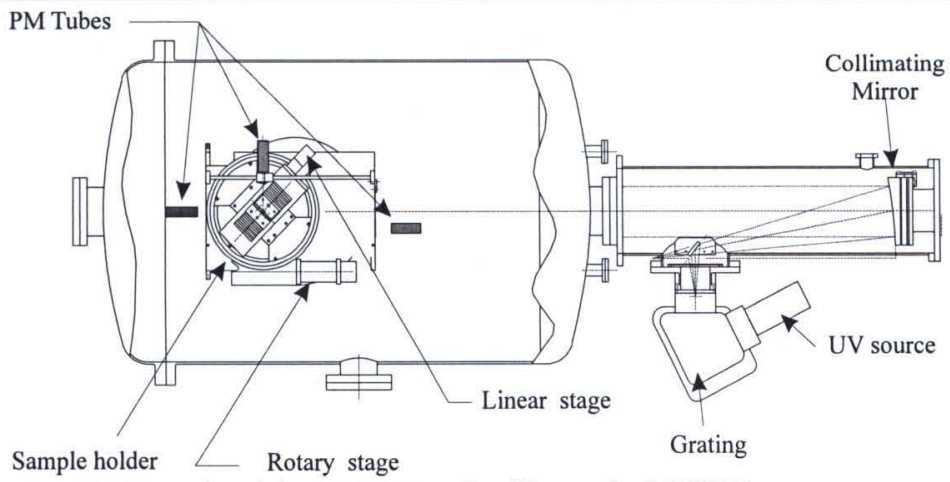




\section{TVLS POLARIZATION MEASUREMENTS}

Although the original theory for spherical-varied-line-space (SVLS) gratings was developed over two decades ago ${ }^{12}$, the actual manufacturing of such gratings is a recent development. These devices are very useful in instruments that must minimize the number of optical elements to improve overall efficiency, since they act as high-resolution wavelength filters and as imaging optics for the UV spectrograph. This is certainly important for the SUMI program where each optical element represents a loss in throughput, especially for the CIV measurement. The toroidal variable-line-space (TVLS) grating is an extension of this technology which allows us to adjust the shape in both the wavelength and spatial axes to further correct for optical aberrations. The TVLS concept is described by Thomas (2003) ${ }^{13}$ and by Poletto \& Thomas $(2004)^{14}$.

In SUMI, a toroidal variable-line-space grating is required for each of the linear polarization channels $(\mathrm{I}+\mathrm{Q}$ and $\mathrm{I}-\mathrm{Q})$ exiting the double Wollaston analyzer (Figure 2). The minimum performance requirements for the spectrograph are listed in Table 1. These goals are based on the filtergraph approximation to magnetic field measurements, which requires $>5$ polarization measurements across a spectral line. Since the width of the line is $\sim 200 \mathrm{~m} \AA$ for CIV and $\sim 400 \mathrm{~m} \AA$ for MgII, the minimum resolutions are $50 \mathrm{~m} \AA$ for CIV and $80 \mathrm{~m} \AA$ for $\mathrm{MgII}$.

To optimize the instrument's imaging performance while fitting it into a sounding rocket payload, the spectrograph was designed to measure CIV in $2^{\text {nd }}$ order and MgII in $1^{\text {st }}$ order. The two TVLS gratings each have the same ruling variations as a function of position across their surface, differing by about $2 \%$ around the central groove density of 2.440 lines per $\mu \mathrm{m}$. These ruling variations can be described by a third-order polynomial with horizontal distance $(y)$ on the grating blank (in $\mathrm{mm}$ ):

$$
\rho(y)=\frac{1}{\frac{1}{\rho_{0}}+\alpha y+\beta y^{2}+\gamma y^{3}} \quad \text { (Zemax equation for VLS ruling) }
$$

where $\rho(y)$ is the ruling as a function of distance from center of the grating, and $\rho_{0}$ is the central ruling frequency (lines $/ \mu \mathrm{m})$. The ruling parameters for the SUMI gratings are: $\rho_{0}=2.440, \alpha=-1.2755 e-4, \beta=1.182 e-9$ and $\gamma=2.4729 e-11$. Both gratings have the same concave toroidal radii of curvature: $1500.000 \mathrm{~mm}$ in the dispersion axis and $1601.792 \mathrm{~mm}$ in the spatial direction. With this design, the spectrometer's wavelength resolution is significantly improved.

\subsection{Polarization test setup}

Figure 8 shows the test setup for the TVLS polarization measurements. The input beam is polarized by a Rochon prism, and the extraordinary beam blocked by a stop. The Rochon prism is mounted on a rotary stage to control polarization angle. An aluminum $/ \mathrm{MgF}_{2}$ mirror of known reflectivity is used to direct the beam to the gratings. The grating is mounted on a rotary stage to control the incident angle; the two gratings on SUMI see slightly different incident angles $\left(17.45^{\circ}\right.$ and $18.5^{\circ}$ ) and the reflectivity is measured at both angles. The aluminum fold mirror can be moved out of the beam to measure the raw beam strength.

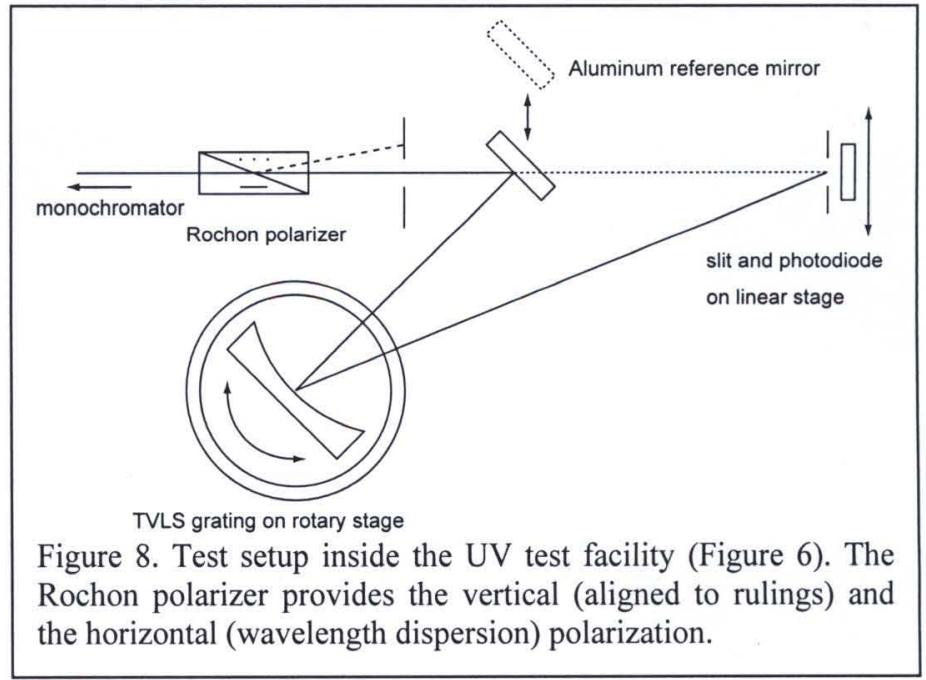


While the grating is concave, its focal length is much longer than the grating-slit distance in this setup and therefore there is no significant convergence or divergence of the beam due to reflection angle effects. Nevertheless, the monochromator's $\sim 4 \mathrm{~nm}$ bandpass, dispersed by the TVLS grating, results in a beam larger than the $10 \mathrm{~mm}$ size of the photodiode. Because the bare photodiode has a poorly defined aperture, a $2.4 \mathrm{~mm}$ aluminum slit is added to the photodiode and the slit/photodiode is moved across the beam in $0.6 \mathrm{~mm}$ increments. The flux is summed to obtain the integrated flux.

\subsection{Polarization measurements}

The SUMI gratings were delivered and tested in the fall of 2004 (reference as 2005 VLS in Figure 9 for SPIE 2005 paper $\left.^{4}\right)$. Unfortunately, the rulings did not meet SUMI's design requirements (Figure 9) and had to be re-ruled. While verifying that the ruling equation from the optical design code (Zemax) was correctly converted to the equation required by the holographic test setup, polarization measurements were made on those gratings in order to develop the test setup (\$4.1) and to study the polarization sensitivity of TVLS gratings. Figure 10 shows the measurements on one of the 2005 gratings. The tested grating shows a $13 \%$ reflectivity for horizontal polarized light at $155 \mathrm{~nm}$, and $23 \%$ for vertical polarization. The data also showed a measurable difference in the reflectivity with only $1^{\circ}$ change in the incident angle $e^{4}$.

All of the gratings measured in 2005 had an

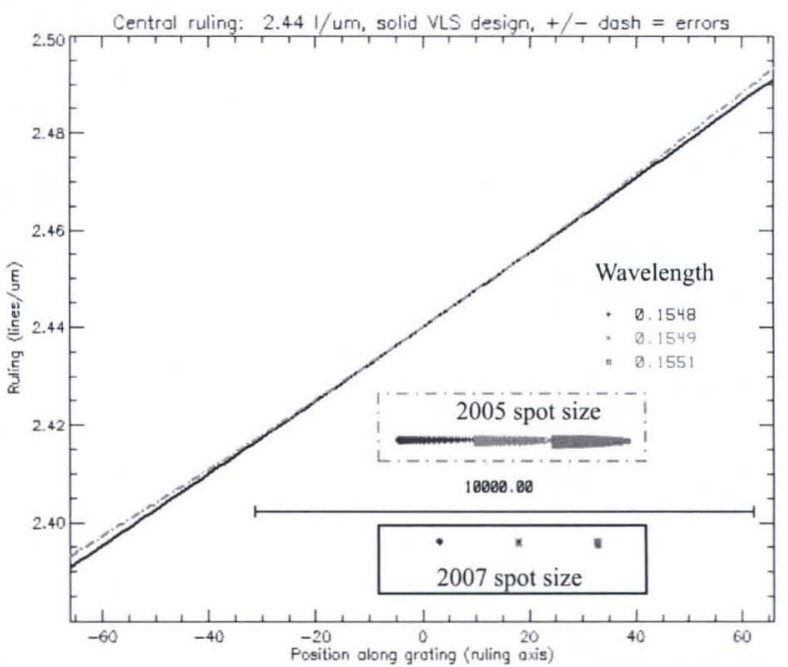

Figure 9. 2007 VLS ruling (black line, Zemax parameters: $\alpha=-1.2755 e-4, \beta=1.182 e-9$ and $\gamma=2.4729 e-11$.) and the 2005 VLS (red dashed line, $\alpha=-1.2714 e-4, \beta=-8.8364 e-8$ and $\gamma=1.555 e-11)$.

aluminum $/ \mathrm{MgF}_{2}$ coating. Based on discussions with

the manufacture (Jobin Yvon), the large differences in the polarization sensitivity were assumed to be related to Wood's anomaly and associated with the $\mathrm{MgF}_{2}$ protective coating. A summary of our 2005 polarization measurements at our primary wavelengths (CIV and MgII) is shown in Table 3. When the gratings were re-ruled, four were coated with just aluminum and two with aluminum/ $\mathrm{MgF}_{2}$. Also an attempt to control the chemical etching was made to improve the blaze angle reflectivity. After completing our initial polarization measurements, Grating 3 (which was originally coated with bare aluminum; see Table 2) was recoated with aluminum/ $\mathrm{MgF}_{2}$ to improve its MgII HP sensitivity ( $\left.9 \%\right)$. The only significant change in the 2005 and 2007 measurements is the reduced reflectivity in the horizontal polarization at the $\mathrm{MgII}$ wavelength for the bare aluminum gratings. Figure 11 (aluminum/ $\mathrm{MgF}_{2}$ ) and Figure 12 (aluminum only) shows two examples of the polarization reflectivity measurements at CIV and MgII. Certainly the reduce horizontal polarization reflectivity of the aluminum grating at MgII is obvious in Figure 12. Figure 12 also shows the typical oxidation loss of a bare aluminum coating at our CIV wavelength.

Table 2. Polarization reflectivity measurements for the six gratings fabricated by Jobin Yvon for SUMI: 2007 measurements. The gratings selected for the first flight are \#1 for the HP and \#3 for the VP.

\begin{tabular}{|c|c|c|c|c|c|}
\hline \multirow[b]{2}{*}{ Grating number } & \multirow{2}{*}{ Coating } & \multicolumn{2}{|c|}{ Horizontal Polarization (HP) } & \multicolumn{2}{|c|}{ Vertical Polarization (VP) } \\
\hline & & CIV ( $2^{\text {nd }}$ order $)$ & $\operatorname{MgII}\left(1^{\text {st }}\right.$ order $)$ & CIV ( $2^{\text {nd }}$ order $)$ & $\mathrm{MgII}\left(1^{\mathrm{st}}\right.$ order $)$ \\
\hline 1. CY11S* & $\mathrm{AL} / \mathrm{MgF}_{2}$ & $12 \%$ & $39 \%$ & $29 \%$ & $52 \%$ \\
\hline 2. CY11O & $\mathrm{AL} / \mathrm{MgF}_{2}$ & $11 \%$ & $33 \%$ & $15 \%$ & $38 \%$ \\
\hline 3. CY11N* & $\mathrm{AL} / \mathrm{MgF}_{2}(A L)$ & $12 \%(16 \%)$ & $41 \%(9 \%)$ & $30 \%(17 \%)$ & $54 \%(49 \%)$ \\
\hline 4. CY11P & $\mathrm{AL}$ & $16 \%$ & $11 \%$ & $18 \%$ & $52 \%$ \\
\hline 5. CY11R & $\mathrm{AL}$ & $12 \%$ & $15 \%$ & $20 \%$ & $62 \%$ \\
\hline 6. CY11M & $\mathrm{AL}$ & $14 \%$ & $16 \%$ & $17 \%$ & $61 \%$ \\
\hline
\end{tabular}

* Acton $\mathrm{AL} / \mathrm{MgF}_{2}$ coatings. Grating 3 was recoated by Acton after the aluminum measurements showed a low HP reflectance MgII ( $9 \%)$ 
Table 3. Polarization reflectivity measurements for the four gratings fabricated by JY for SUMI: 2005 measurements

\begin{tabular}{|c|c|c|c|c|c|}
\hline \multirow{2}{*}{ Grating number } & \multirow{2}{*}{ Coating } & \multicolumn{2}{|c|}{ Horizontal Polarization (HP) } & \multicolumn{2}{c|}{ Vertical Polarization (VP) } \\
\cline { 3 - 6 } & & $\mathrm{CIV}\left(2^{\text {nd }}\right.$ order $)$ & $\mathrm{MgII}\left(1^{\text {st }}\right.$ order $)$ & $\mathrm{CIV}\left(2^{\text {nd }}\right.$ order $)$ & $\mathrm{MgII}\left(1^{\text {st }}\right.$ order $)$ \\
\hline 1. B37J & $\mathrm{AL} / \mathrm{MgF}_{2}$ & $12 \%$ & $38 \%$ & $19.5 \%$ & $58 \%$ \\
\hline $2 . \mathrm{B} 43 \mathrm{Q}$ & $\mathrm{AL} / \mathrm{MgF}_{2}$ & $13 \%$ & $34 \%$ & $16 \%$ & $68 \%$ \\
\hline $3 . \mathrm{B} 43 \mathrm{P}$ & $\mathrm{AL} / \mathrm{MgF}_{2}$ & $12 \%$ & $38 \%$ & $19 \%$ & $75 \%$ \\
\hline $4 . \mathrm{B} 43 \mathrm{O}$ & $\mathrm{AL} / \mathrm{MgF}_{2}$ & $13 \%$ & $26 \%$ & $20.5 \%$ & $68 \%$ \\
\hline
\end{tabular}
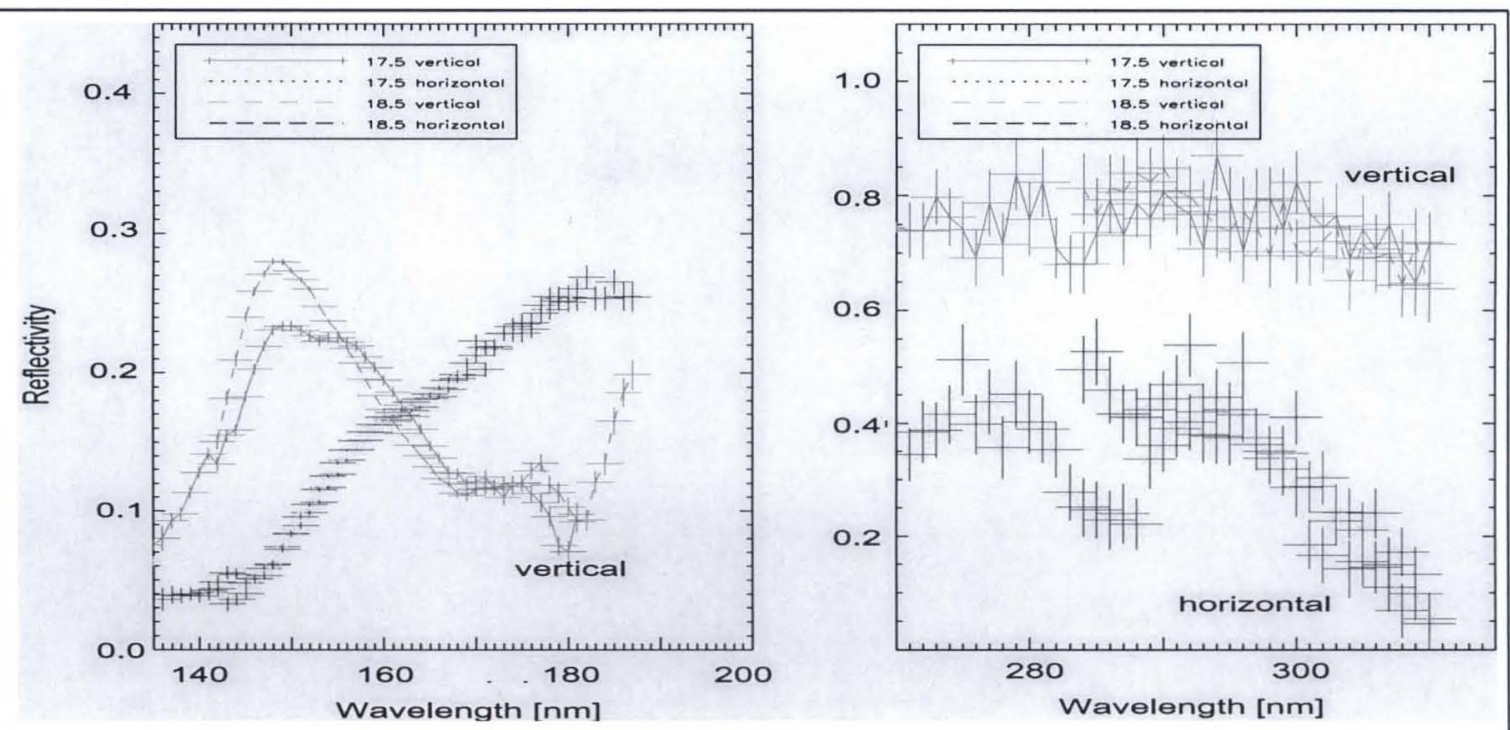

Figure 10. Polarization reflectivity made on 2005 TVLS gratings (red line in Figure 9).
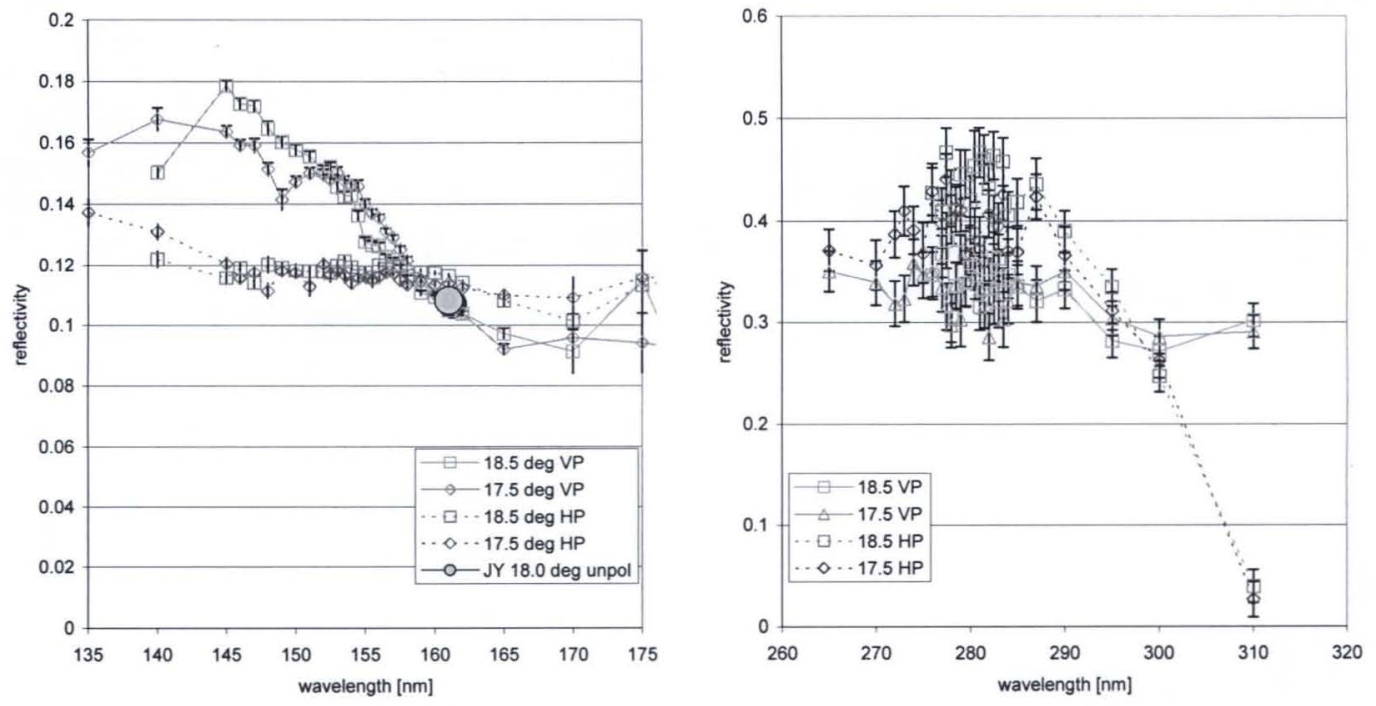

Figure 11. Polarization reflectivity measurements on TVLS grating 2 with $\mathrm{JY}$ aluminum $/ \mathrm{MgF}_{2}$ 

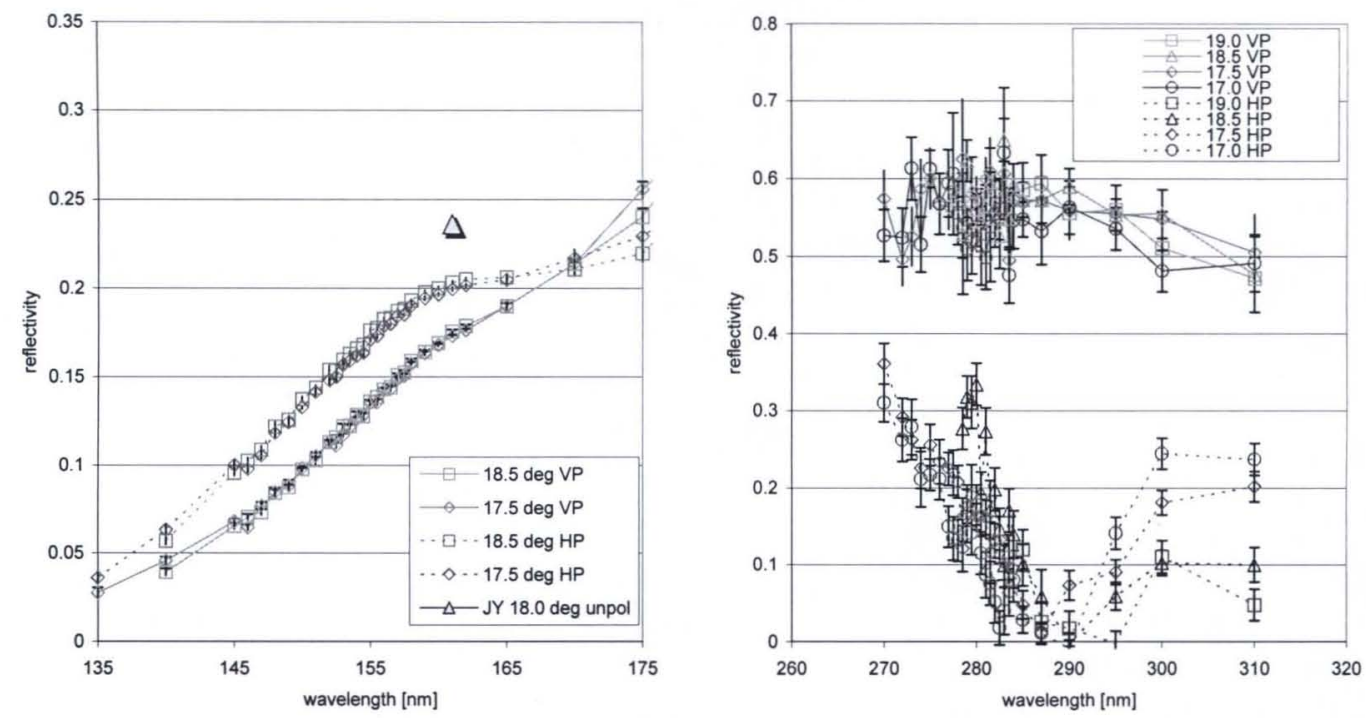

Figure 12. Polarization reflectivity measurements on TVLS grating 6 with bare aluminum coating
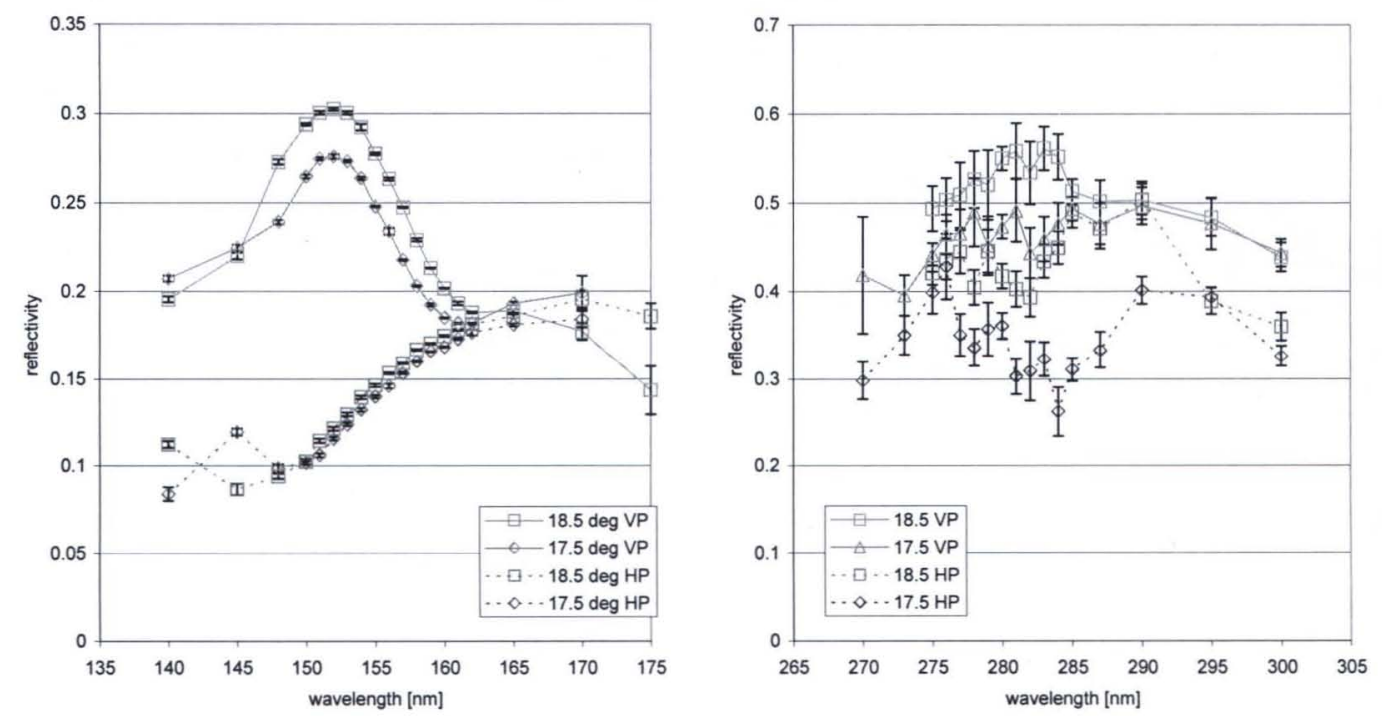

Figure 13. Polarization reflectivity vs. wavelength on TVLS grating 3 with Acton aluminum/ $\mathrm{MgF}_{2}$ coating 6682-32 V. 2 (p.10 of 12) / Color: No / Format: Letter / Date: 8/9/2007 10:18:56 AM 


\section{SUMMARY}

During our TVLS grating tests other anomalies (Figures 13 and 14) in the polarization reflectance measurements were noted that indicates that Wood's anomalies still exists in our gratings, although how or if the $\mathrm{MgF}_{2}$ protective coating is creating them will require additional measurements and modeling. The large difference in the polarization reflectance for the aluminum gratings at MgII, limit them to the vertical polarization (slit orientation) measurements. Based on our 2007 measurements, gratings 1 and 3 have been selected for SUMI's initial flight. Unfortunately SUMI's launch schedule forced us to limit our research program to our primary wavelengths but we hope to extend our polarization measurements to cover the 130 to $300 \mathrm{~nm}$ wavelength range looking for anomalies in both the first and second order wavelengths and to compare those measurements with grating models such as PCgrate.

Finally, all of the optical components for SUMI have been measured and meet the instrument requirements. The major mechanical components have been designed and fabricated and the payload is being assembled. Although some optical testing has been delayed due to reduce funding for $\mathrm{FY} 07$, the final system alignment and vibration tests are expected to be completed next spring with a launch date set for the June 2008 .
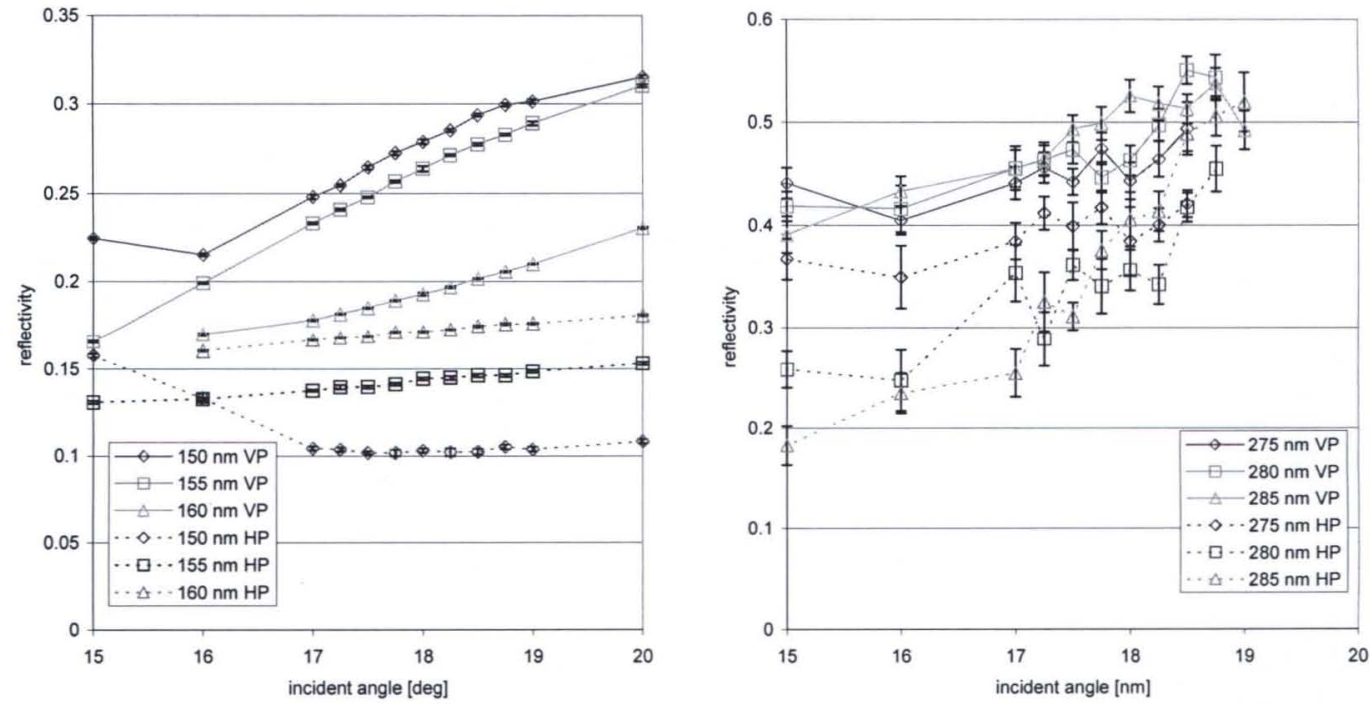

Figure 14. Polarization reflectivity vs. incident angle, TVLS grating 3 with Acton $\mathrm{MgF}_{2}$ coating 


\section{REFERENCES}

1. West, E. A., J. G. Porter, J. M. Davis, G. A. Gary, D. M. Rabin, R. J. Thomas, and J. M. Davila, "Overview of the Solar Ultraviolet Magnetograph Investigation," SPIE,Vol. 4139, pp. 350-361 (2000).

2. West, E. A., J. G. Porter, J. M. Davis, G. A. Gary, and M. Adams, "Development of a polarimeter for magnetic field measurements in the ultraviolet," SPIE Vol. 4481, pp. 109-117 (2001).

3. West, E. A., J. G. Porter, J. M. Davis, G. A. Gary, M. Adams, S. Smith and J. F. Hraba, "Optical characteristics of the Marshall Space Flight Center Solar Ultraviolet Magnetograph,” SPIE Vol. 4498, pp. 101-110 (2001).

4. K. Kobayashi, E. A. West and M. Noble, "Polarization measurements in the Vacuum Ultraviolet," Polarization Science and Remote Sensing II, Proc SPIE, 5888, p. 58880G-1 to -12, 2005.

5. E. A. West, K. Kobayashi, J. M. Davis and G. A. Gary, "The Solar Ultraviolet Magnetograph Investigation Sounding Rocket Program," Solar Physics and Space Weather Instrumentation II, Proc SPIE 6689-6, 2007.

6. Moore, Davis, and Hathaway, "High-Resolution Solar Magnetography from Space: Beyond Solar-B," http://solarscience.msfc.nasa.gov/Beyond_Solar-B.shtml, 2001

7. J. Davis, E. West, R. Moore, G. Gary K. Kobayashi, J. Oberright, D. Evans, H. Wood, J. L. R. Saba and D. Alexander, "MTRAP: The Magnetic Transition Region Probe," Solar Physics and Space Weather Instrumentation, Proc. SPIE, 5901, p. 590112-1 to -8, 2005.

8. G. A. Gary, "Plasma Beta above a Solar Active Region: Rethinking the Paradigm," Solar Phys., 203, 71, 2001.

9. Gurman, J. B., “The MG II H line in sunspot umbrae," Solar Phys., 90, 13-15, 1984.

10. H. Uitenbroek, "Multilevel Radiative Transfer with Partial Frequency Redistribution," Astrophysical Journal, 557, 389-398, 2001.

11. H. Peter, "On the nature of the transition region from the chromosphere to the corona of the Sun," $A \& A, 374,1108$ $1120,2001$.

12. T. Kita and T. Harada, "Use of Aberration-Corrected Concave Gratings in Optical Demultiplexers," Applied Optics, 22, 819-825, 1983.

13. R. J. Thomas, "Toroidal varied line-space (TVLS) gratings," Proc SPIE, 4853, 411-418, 2003.

14. L. Poletto and R. J. Thomas, "Stigmatic Spectrometers for Extended Sources: Design with Toroidal Varied LineSpace (TVLS) Gratings," Applied Optics, 43, 2029-2038, 2004. 